\title{
NOTICE
}

\section{Guest Investigators at the Mount Wilson and Palomar Observatories}

$T^{1}$ HE Mount Wilson and Palomar Observatories, following a practice of many years, will continue to make observing facilities available to qualified guest investigators from other institutions. The Observatories are operated jointly by the Carnegie Institution of Washington and the California Institute of Technology.

Interested astronomers may obtain a leaflet entitled Information for Guest Investigators at the Mount Wilson and Palomar Observatories by addressing the Office of the Director, 813 Santa Barbara Street, Pasadena, California, 91106.

It is requested that an explicit research proposal be submitted by the prospective observer. Approval of observing programs and allotment of time on the telescopes will be made on the basis of scientific merit of the proposal and suitability of the available instruments.

It is desirable that research proposals and requests for guest investigator privileges for a given calendar year be received by October 31 of the preceding year. Demands for observing time are such that allotments can seldom. be made on shorter notice.

H. W. BABCOCK 